\title{
CHARACTERIZATIONS OF THE GELFAND-SHILOV SPACES VIA FOURIER TRANSFORMS
}

\author{
JAEYOUNG CHUNG, SOON-YEONG CHUNG, AND DOHAN KIM
}

(Communicated by Palle E. T. Jorgensen)

\begin{abstract}
We give symmetric characterizations, with respect to the Fourier transformation, of the Gelfand-Shilov spaces of (generalized) type $S$ and type $W$. These results explain more clearly the invariance of these spaces under the Fourier transformations.
\end{abstract}

\section{INTRODUCTION}

The purpose of this paper is to give new characterizations of the Gelfand-Shilov spaces of (generalized) type $S$ and type $W$ by means of the Fourier transformation. Gelfand and Shilov introduced the above spaces in [6] to study the uniqueness of the Cauchy problems of partial differential equations. In [1] the Schwartz space $\mathcal{S}$ is characterized by the estimates

$$
\sup _{x}\left|x^{\alpha} \varphi(x)\right|<\infty, \quad \sup _{x}\left|\partial^{\beta} \varphi(x)\right|<\infty,
$$

or by the estimates

$$
\sup _{x}\left|x^{\alpha} \varphi(x)\right|<\infty, \quad \sup _{\xi}\left|\xi^{\beta} \hat{\varphi}(\xi)\right|<\infty,
$$

where the Fourier transform $\hat{\varphi}$ is defined by $\hat{\varphi}(\xi)=\int e^{-i x \cdot \xi} \varphi(x) d x$.

In addition, the Sato space $\mathcal{F}$ of test functions for the Fourier hyperfunctions is characterized by the estimates

$$
\sup _{x}|\varphi(x)| \exp k|x|<\infty, \quad \sup _{\xi}|\hat{\varphi}(\xi)| \exp h|\xi|<\infty
$$

for some $h, k>0$ in [2].

Generalizing the above results in a similar manner we give more symmetric characterizations of the Gelfand-Shilov spaces in terms of the Fourier transformations as follows.

I. For the space $S_{r}^{s}$ the following statements are equivalent.

(1) $\varphi \in S_{r}^{s}$;

(2) $\sup _{x}|\varphi(x)| \exp k|x|^{1 / r}<\infty, \quad \sup _{\xi}|\hat{\varphi}(\xi)| \exp h|\xi|^{1 / s}<\infty$ for some $h, k>0$.

Received by the editors November 3, 1994 and, in revised form, January 26, 1995.

1991 Mathematics Subject Classification. Primary 46F12, 46F15.

Key words and phrases. Gelfand-Shilov spaces, Fourier transform, associated function.

Partially supported by Korea Research Foundation and GARC.

(C)1996 American Mathematical Society 
II. For the space $S_{M_{p}}^{N_{p}}$ the following statements are equivalent.

(1) $\varphi \in S_{M_{p}}^{N_{p}}$;

(2) $\sup _{x}|\varphi(x)| \exp M(a x)<\infty, \quad \sup _{\xi}|\hat{\varphi}(\xi)| \exp N(b \xi)<\infty$ for some $a, b>0$, where $M(x)$ and $N(\xi)$ are associated functions of $M_{p}$ and $N_{p}$, respectively (see (2.1) for the definition).

III. For the space $W_{M}^{\Omega}$ the following statements are equivalent.

(1) $\varphi \in W_{M}^{\Omega}$;

(2) $\sup _{x}|\varphi(x)| \exp M(a|x|)<\infty, \quad \sup _{\xi}|\hat{\varphi}(\xi)| \exp \Omega^{*}(b|\xi|)<\infty$ for some $a, b>$ 0 , where $\Omega^{*}$ is the Young conjugate of $\Omega$ (see Definition 3.3).

\section{Characterization of Gelfand-Shilov SPACES OF (GENERALIZED) TYPE S}

In this section we characterize the Gelfand-Shilov spaces of type $S$ and generalized type $S$ in a more symmetric way by means of the Fourier transformation, which are generalizations of (1.2) and (1.3).

We first introduce the Gelfand-Shilov spaces of generalized type $S$ and type $S$. Let $M_{p}, p=0,1,2, \ldots$, be a sequence of positive numbers. We impose the following conditions on $M_{p}$ :

(M.1) (logarithmic convexity) $M_{p}^{2} \leq M_{p-1} M_{p+1}, \quad p=1,2, \ldots$;

(M.2) (stability under differential operators) there are constants $A$ and $H$ such that $M_{p+q} \leq A H^{p+q} M_{p} M_{q}, p, q=0,1,2, \ldots$

Definition 2.1. Let $M_{p}$ and $N_{p}, p=0,1,2, \ldots$, be sequences of positive numbers. Then the Gelfand-Shilov spaces $S_{M_{p}}, S^{N_{p}}$ and $S_{M_{p}}^{N_{p}}$ consist of all infinitely differentiable functions $\varphi(x)$ on $\mathbb{R}^{n}$ satisfying the following estimates, respectively,

$$
\begin{aligned}
& \sup _{x}\left|x^{\alpha} \partial^{\beta} \varphi(x)\right| \leq C_{\beta} A^{|\alpha|} M_{|\alpha|}, \\
& \sup _{x}\left|x^{\alpha} \partial^{\beta} \varphi(x)\right| \leq C_{\alpha} B^{|\beta|} N_{|\beta|}, \\
& \sup _{x}\left|x^{\alpha} \partial^{\beta} \varphi(x)\right| \leq C A^{|\alpha|} B^{|\beta|} M_{|\alpha|} N_{|\beta|}
\end{aligned}
$$

for some positive constants $A$ and $B$ for all multi-indices $\alpha$ and $\beta$.

Remark. In particular, if $M_{p}=p !^{r}$ and $N_{p}=p !^{s}$, then we denote the spaces $S_{M_{p}}$, $S^{N_{p}}$ and $S_{M_{p}}^{N_{p}}$ by $S_{r}, S^{s}$ and $S_{r}^{s}$, respectively, and call these spaces the GelfandShilov spaces of type $S$.

Definition 2.2. Let $M_{p}$ and $N_{p}$ be sequences of positive numbers satisfying (M.1). Then we write $M_{p} \subset N_{p}\left(M_{p} \prec N_{p}\right.$, respectively) if there are constants $L, C>0$ (for any $L>0$ there is a constant $C>0$, respectively) such that $M_{p} \leq C L^{p} N_{p}, \quad p=$ $0,1,2, \ldots$.

Also, $M_{p}$ and $N_{p}$ are said to be equivalent if $M_{p} \subset N_{p}$ and $M_{p} \supset N_{p}$ hold.

Theorem 2.3. If $M_{p}$ and $N_{p}$ satisfy (M.1) and (M.2) and $M_{p} N_{p} \supset p$ !, then the following conditions are equivalent.

(i) $\varphi \in S_{M_{p}}^{N_{p}}$.

(ii) There exist positive constants $A, B$ and $C$ such that

$$
\sup _{x}\left|x^{\alpha} \varphi(x)\right| \leq C A^{|\alpha|} M_{|\alpha|}, \quad \sup _{x}\left|\partial^{\beta} \varphi(x)\right| \leq C B^{|\beta|} N_{|\beta|}
$$

for all multi-indices $\alpha$ and $\beta$. 
(iii) There exist positive constants $A, B$ and $C$ such that

$$
\sup _{x}\left|x^{\alpha} \varphi(x)\right| \leq C A^{|\alpha|} M_{|\alpha|}, \quad \sup _{\xi}\left|\xi^{\beta} \hat{\varphi}(\xi)\right| \leq C B^{|\beta|} N_{|\beta|}
$$

for all multi-indices $\alpha$ and $\beta$.

Proof. The implications (i) $\Rightarrow$ (ii) and (i) $\Rightarrow$ (iii) follow immediately from the equality $\widehat{S_{M_{p}}^{N_{p}}}=S_{N_{p}}^{M_{p}}$. We now prove the implication (ii) $\Rightarrow$ (i). We can use the $L^{2}$-norm instead of the supremum norm. Using integration by parts, the Leibniz formula and the Schwarz inequality we obtain

$$
\begin{aligned}
\left\|x^{\alpha} \partial^{\beta} \varphi(x)\right\|_{L^{2}}^{2} & =\int_{\mathbb{R}^{n}}\left[x^{2 \alpha} \partial^{\beta} \varphi(x)\right] \partial^{\beta} \varphi(x) d x \\
& \leq \sum_{\substack{\gamma \leq 2 \alpha \\
\gamma \leq \beta}}\left(\begin{array}{c}
\beta \\
\gamma
\end{array}\right)\left(\begin{array}{c}
2 \alpha \\
\gamma
\end{array}\right) \gamma !\left\|\partial^{2 \beta-\gamma} \varphi(x)\right\|_{L^{2}}\left\|x^{2 \alpha-\gamma} \varphi(x)\right\|_{L^{2}} \\
& \leq C^{2} \sum_{\gamma}\left(\begin{array}{c}
\beta \\
\gamma
\end{array}\right)\left(\begin{array}{c}
2 \alpha \\
\gamma
\end{array}\right) \gamma ! A^{2(|\alpha|+|\beta|-|\gamma|)} M_{|2 \alpha-\gamma|} N_{|2 \beta-\gamma|} .
\end{aligned}
$$

It follows from the conditions (M.1), (M.2) and $M_{p} N_{p} \supset p$ ! that

$$
\begin{aligned}
\left\|x^{\alpha} \partial^{\beta} \varphi(x)\right\|_{L^{2}}^{2} & \leq C^{2} A^{2(|\alpha|+|\beta|)} M_{|2 \alpha|} N_{|2 \beta|} \sum_{\gamma}\left(\begin{array}{c}
\beta \\
\gamma
\end{array}\right)\left(\begin{array}{c}
2 \alpha \\
\gamma
\end{array}\right) \gamma ! /\left(M_{|\gamma|} N_{|\gamma|}\right) \\
& \leq C^{2}(2 A H)^{2(|\alpha|+|\beta|)} M_{|\alpha|}^{2} N_{|\beta|}^{2},
\end{aligned}
$$

which implies that $\varphi(x)$ belongs to $S_{M_{p}}^{N_{p}}$. Therefore, it remains to prove (iii) $\Rightarrow$ (ii). The inequality $\left|\xi^{\beta} \hat{\varphi}(\xi)\right| \leq C B^{|\beta|} N_{|\beta|}$ means that

$$
|\hat{\varphi}(\xi)| \leq C \exp [-N(|\xi| / B)]
$$

where $N(\rho)$ is the associated function of $N_{p}$ defined by

$$
N(\rho)=\sup _{p} \log \frac{\rho^{p}}{N_{p}} .
$$

Therefore, by the conditions (M.1) and (M.2) of $N_{p}$ we have

$$
\begin{aligned}
\left|\partial^{\beta} \varphi(x)\right| & \leq(2 \pi)^{-n} \int\left|e^{i x \cdot \xi} \xi^{\beta} \hat{\varphi}(\xi)\right| d \xi \\
& \leq C_{1} \int|\xi|^{|\beta|} \exp [-N(|\xi| / B)] d \xi \\
& \leq C_{1} \sup _{\xi}\left[|\xi|^{2|\beta|} \exp (-N(|\xi| / B))\right]^{1 / 2} \int \exp [-N(|\xi| / B) / 2] d \xi \\
& \leq C_{2} B^{|\beta|} N_{2|\beta|}^{1 / 2} \leq C_{2} B_{1}^{|\beta|} N_{|\beta|},
\end{aligned}
$$

which completes the proof.

Using the associated function as in (2.1) we can characterize $S_{M_{p}}^{N_{p}}$ as follows. 
Corollary 2.4. If $M_{p}$ and $N_{p}$ satisfy (M.1) and (M.2) and $M_{p} N_{p} \supset p$ !, then the following conditions are equivalent.

(i) $\varphi \in S_{M_{p}}^{N_{p}}$.

(ii) There exist positive constants $a$ and $b$ such that

$$
\sup _{x}|\varphi(x)| \exp M(a x)<\infty, \quad \sup _{\xi}|\hat{\varphi}(\xi)| \exp N(b \xi)<\infty .
$$

In particular, putting $M_{p}=p !^{r}$ and $N_{p}=p !^{s}$ we can give simple characterizations for the Gelfand-Shilov spaces of type $\mathrm{S}$ as corollaries.

Corollary 2.5. If $r+s \geq 1$, then the following are equivalent:

(i) $\varphi \in S_{r}^{s}$.

(ii) There exist positive constants $h$ and $k$ such that

$$
\sup _{x}|\varphi(x)| \exp k|x|^{1 / r}<\infty, \quad \sup _{\xi}|\hat{\varphi}(\xi)| \exp h|\xi|^{1 / s}<\infty .
$$

Remark. We can easily prove the similar results on the characterization of $S_{M_{p}}$ and $S^{N_{p}}$.

\section{Characterization of Gelfand-Shilov spaces of type $W$}

In this section we characterize the Gelfand-Shilov spaces of type $W$ in a more symmetric way by means of the Fourier transformation.

Let $M(x)$ and $\Omega(y)$ be differentiable functions on $[0, \infty)$ satisfying the condition $(\mathrm{K}): M(0)=\Omega(0)=M^{\prime}(0)=\Omega^{\prime}(0)=0$ and their derivatives are continuous, increasing and tending to infinity.

We now define the Gelfand-Shilov spaces of type $W$ as in [6].

Definition 3.1. (i) The space $W_{M}$ consists of all infinitely differentiable functions $\varphi(x)$ on $\mathbb{R}^{n}$ satisfying the estimate $\left|\partial^{\beta} \varphi(x)\right| \leq C_{\beta} \exp (-M(a|x|))$ for some $a>0$.

(ii) The space $W^{\Omega}$ consists of all entire analytic functions $\varphi(\zeta)$ on $\mathbb{C}^{n}$ satisfying the estimate $\left|\zeta^{\alpha} \varphi(\zeta)\right| \leq C_{\alpha} \exp \Omega(b|\eta|)$ for some $b>0$, where $\zeta=\xi+i \eta \in \mathbb{R}^{n}+i \mathbb{R}^{n}$.

(iii) The space $W_{M}^{\Omega}$ consists of all entire analytic functions $\varphi(\zeta)$ on $\mathbb{C}^{n}$ satisfying the estimate $|\varphi(\xi+i \eta)| \leq C \exp (-M(a|\xi|)+\Omega(b|\eta|))$ for some $a, b>0$.

In order to relate the sequences $M_{p}$ and the functions $M(x)$ we need the following definitions.

Definition 3.2. If $M(\rho)$ is an increasing convex function in $\log \rho$ and increases more rapidly than $\log \rho^{p}$ for any $p$ as $\rho$ tends to infinity, we define its defining sequence by

$$
M_{p}=\sup _{\rho>0} \rho^{p} / \exp M(\rho), \quad p=0,1,2, \ldots
$$

Definition 3.3. Let $M:[0, \infty) \rightarrow[0, \infty)$ be a convex and increasing function with $M(0)=0$ and $\lim _{x \rightarrow \infty} x / M(x)=0$. Then we define its Young conjugate $M^{*}$ by $M^{*}(\rho)=\sup _{x}(x \rho-M(x))$.

To prove the main theorem on the characterizations of the Gelfand-Shilov spaces of type $W$ we need the following relations between the defining sequences and the associated functions as in $[3,5,9]$. 
Proposition 3.4 ([9]). If $M_{p}$ satisfies (M.1), then $M_{p}$ is the defining sequence of the associated function of itself.

Proposition 3.5 ([5]). Let $M:[0, \infty) \rightarrow[0, \infty)$ be a function satisfying the condition $(\mathrm{K})$. Then $M(x)$ is equivalent to the associated function of the defining sequence of itself.

Here, $M(x)$ and $N(x)$ are said to be equivalent if there exist constants $A$ and $B$ such that $M(A x) \leq N(x) \leq M(B x)$.

Proposition 3.6 ([9]). Let $m_{p}=M_{p} / M_{p-1}, p=1,2, \ldots$, and let $m(\lambda)$ be the number of $m_{p}$ such that $m_{p} \leq \lambda$. Then we have $M(\rho)=\int_{0}^{\rho} m(\lambda) / \lambda d \lambda$.

Lemma 3.7. Let $M(\rho)$ be a function satisfying the condition $(\mathrm{K})$. Then the defining sequence $M_{p}^{*}$ of the Young conjugate $M^{*}(\rho)$ of $M(\rho)$ is equivalent to $p ! / M_{p}$, where $M_{p}$ is the defining sequence of $M(\rho)$. In fact, $M_{p}^{*}=(p / e)^{p} / M_{p}$. Consequently, $M_{p}$ satisfies the following conditions:

(M.1) ${ }^{\prime}$ (strong logarithmic convexity) $m_{p}=M_{p} / M_{p-1}$ is increasing and tends to infinity as $p \rightarrow \infty$;

(M.1)* (duality) $p ! / M_{p}$ satisfies (M.1)'.

Conversely, if $M_{p}$ satisfies (M.1)' and (M.1)*, then the associated function $M^{\#}(\rho)$ of $p ! / M_{p}$ and the Young conjugate $M^{*}(\rho)$ of the associated function $M(\rho)$ of $M_{p}$ are equivalent.

Proof. We may assume that $M^{\prime}(\rho)$ is strictly increasing. Then it is easy to see that $M^{*}(\rho)=\int_{0}^{\rho} M^{\prime-1}(t) d t$ where $M^{\prime-1}$ is the inverse function of $M^{\prime}$. Let $g(t)=t^{p} / \exp M^{*}(t)$. To find the maximum of $g(t)$ for $t>0$, taking logarithm, differentiating and equating the result to zero, we obtain

$$
p / t=M^{\prime-1}(t) .
$$

Let $t_{0}$ be the root of the equation (3.1). Then there exists $\rho_{0}>0$ such that $M^{\prime}\left(\rho_{0}\right)=t_{0}$. Putting $t=M^{\prime}\left(\rho_{0}\right)$ in (3.1) we have $\rho_{0} M^{\prime}\left(\rho_{0}\right)=p$. Thus we have

$$
M^{*}\left(M^{\prime}\left(\rho_{0}\right)\right)=\sup _{x}\left(x M^{\prime}\left(\rho_{0}\right)-M(x)\right)=\rho_{0} M^{\prime}\left(\rho_{0}\right)-M\left(\rho_{0}\right)
$$

since the function $h(x)=x M^{\prime}\left(\rho_{0}\right)-M(x)$ takes its maximum at $x=\rho_{0}$. Therefore we have

$$
\begin{aligned}
M_{p}^{*} & =\sup _{t>0} \frac{t^{p}}{\exp M^{*}(t)}=\frac{t_{0}^{p}}{\exp M^{*}\left(t_{0}\right)}=\frac{\left[M^{\prime}\left(\rho_{0}\right)\right]^{p}}{M^{*}\left(M^{\prime}\left(\rho_{0}\right)\right)} \\
& =\frac{\left[M^{\prime}\left(\rho_{0}\right)\right]^{p}}{\exp \left[\rho_{0} M^{\prime}\left(\rho_{0}\right)-M\left(\rho_{0}\right)\right]}=\frac{\left[M^{\prime}\left(\rho_{0}\right)\right]^{p}}{\exp \left(p-M\left(\rho_{0}\right)\right)} \\
& =\left(\frac{p}{e}\right)^{p} \frac{\exp M\left(\rho_{0}\right)}{\rho_{0}^{p}}=\left(\frac{p}{e}\right)^{p} \frac{1}{M_{p}} .
\end{aligned}
$$

For the converse, by Stirling's formula it is easy to see that $p$ ! and $(p / e)^{p}$ are equivalent. So we have for any $t, \rho>0$

$$
M(t)+M^{\#}(\rho)=\sup _{p} \log \frac{t^{p}}{M_{p}}+\sup _{p} \log \frac{\rho^{p} M_{p}}{p !} \geq \sup _{p} \log \frac{(t \rho)^{p}}{p !} \geq A t \rho
$$

for some $A>0$, where $M^{\#}(\rho)$ is the associated functions of $p ! / M_{p}$. 
Thus we have

$$
M^{\#}(\rho) \geq A t \rho-M(t) .
$$

Taking the supremum for $t$ in the right-hand side of (3.2), we have $M^{\#}(\rho) \geq$ $M^{*}(A \rho)$. Since $M_{p}$ satisfies (M.1) ${ }^{\prime}$ and (M.1)*, we may assume that the sequences $m_{p}=M_{p} / M_{p-1}$ and $p / m_{p}$ are strictly increasing.

We denote by $m(\lambda)$ the number of $m_{p}$ such that $m_{p} \leq \lambda$. Then we have by Proposition 3.6

$$
M^{*}(\rho)=\sup _{x} \int_{0}^{x}(\rho-m(\lambda) / \lambda) d \lambda .
$$

Putting $\rho=p / m_{p}$ and $x=m_{p}$, we obtain

$$
\begin{aligned}
M^{*}\left(p / m_{p}\right) & \geq \int_{0}^{m_{p}}\left(p / m_{p}-m(\lambda) / \lambda\right) d \lambda \\
& =p-\int_{0}^{m_{p}} m(\lambda) / \lambda d \lambda \\
& =p-\sum_{j=1}^{p-1} \int_{m_{j}}^{m_{j+1}} j / \lambda d \lambda \\
& =p+\log \frac{m_{1} \cdots m_{p-1}}{m_{p}^{p-1}} .
\end{aligned}
$$

On the other hand, let $m^{\#}(\lambda)$ be the number of $p / m_{p}$ such that $p / m_{p} \leq \lambda$. Then we have

$$
\begin{aligned}
M^{\#}\left(p / m_{p}\right) & =\int_{0}^{p / m_{p}} m^{\#}(\lambda) / \lambda d \lambda \\
& =\sum_{j=1}^{p-1} \int_{j / m_{j}}^{(j+1) / m_{j+1}} j / \lambda d \lambda \\
& =\log \frac{p^{p} m_{1} \cdots m_{p-1}}{p ! \cdot m_{p}^{p-1}} \\
& \leq p+\log \frac{m_{1} \cdots m_{p-1}}{m_{p}^{p-1}} \\
& \leq M^{*}\left(p / m_{p}\right) .
\end{aligned}
$$

Now, for any $\rho>0$ such that $p / m_{p}<\rho<(p+1) / m_{p+1}$ we have

$$
\begin{aligned}
M^{*}(\rho) & \geq M^{*}\left(p / m_{p}\right) \geq M^{\#}\left(p / m_{p}\right) \\
& \geq M^{\#}\left(\frac{1}{2}(p+1) / m_{p+1}\right) \geq M^{\#}\left(\frac{1}{2} \rho\right),
\end{aligned}
$$

which completes the proof.

We are now in a position to state and prove the main theorems on the characterizations of the Gelfand-Shilov spaces of type $W$. 
Theorem 3.8. If there is a constant $L$ such that $M(x) \leq \Omega(L x)$, then the space $W_{M}^{\Omega}$ is characterized by the following estimates

$$
|\varphi(x)| \leq C \exp (-M(a|x|)), \quad|\hat{\varphi}(\xi)| \leq C \exp \left(-\Omega^{*}(b|\xi|)\right) .
$$

Proof. If $\varphi \in C^{\infty}\left(\mathbb{R}^{n}\right)$ satisfies (3.3), then $\varphi(x)$ satisfies

$$
\sup _{x}\left|x^{\alpha} \varphi(x)\right| \leq C A^{|\alpha|} M_{|\alpha|}, \quad \sup _{\xi}\left|\xi^{\beta} \hat{\varphi}(\xi)\right| \leq C B^{|\beta|} N_{|\beta|}
$$

for some $A, B>0$, where $M_{p}$ and $N_{p}$ are the defining sequences of $M(x)$ and $\Omega^{*}(y)$, respectively. Then the sequences $M_{p}$ and $N_{p}$ satisfy (M.1) ${ }^{\prime}$ and (M.1)* by Lemma 3.7 and the condition $M(x) \leq \Omega(L x)$ implies $M_{p} N_{p} \supset p$ !. Therefore $\varphi(x)$ belongs to $S_{M_{p}}^{N_{p}}$ by Theorem 2.3, since (M.1)' and (M.1)* are stronger than (M.1) and (M.2), respectively. We now prove that $S_{M_{p}}^{N_{p}} \subset W_{M}^{\Omega}$. Let $\varphi \in S_{M_{p}}^{N_{p}}$. Then for every $\alpha, \beta \in \mathbb{N}_{0}^{n}$ we obtain

$$
\left|\xi^{\alpha} \partial^{\beta} \varphi(\xi)\right| \leq C A^{|\alpha|} B^{|\beta|} M_{|\alpha|} N_{|\beta|}
$$

for some $A, B>0$. Since $N_{p}$ satisfies (M.1)* or $p ! / N_{p}$ satisfies (M.1)', it is easy to see that $N_{p} \prec p$ !. Hence the function $\varphi(\xi)$ can be continued analytically into the complex domain as an entire analytic function. Applying the Taylor expansion and the inequality (3.4) we have

$$
\begin{aligned}
\left|\xi^{\alpha} \varphi(\xi+i \eta)\right| & \leq \sum_{\gamma \in \mathbb{N}_{0}^{n}} \frac{\left|\xi^{\alpha} \partial^{\gamma} \varphi(\xi)\right|}{\gamma !}|\eta|^{|\gamma|} \\
& \leq C \sum_{\gamma \in \mathbb{N}_{0}^{n}} A^{|\alpha|} M_{|\alpha|} N_{|\gamma|}|B \eta|^{|\gamma|} / \gamma ! \\
& \leq 2^{n} C A^{|\alpha|} M_{|\alpha|} \exp N^{\#}(2 B|\eta|) .
\end{aligned}
$$

Dividing $|\xi|^{|\alpha|}$ in both sides of the inequality (3.5) and taking infimum for $|\alpha|$ in the right-hand side of (3.5), we have

$$
|\varphi(\xi+i \eta)| \leq 2^{n} C \exp \left[-M(|\xi| / A)+N^{\#}(2 B|\eta|)\right] .
$$

Note that we may use $|\xi|^{|\alpha|} \partial^{\beta} \varphi(\xi) \mid$ instead of $\left|\xi^{\alpha} \partial^{\beta} \varphi(\xi)\right|$ in (3.4). Also, Lemma 3.7 implies

$$
N^{\#}(2 B|\eta|) \leq N^{*}\left(B^{\prime}|\eta|\right) \leq\left(\Omega^{*}\right)^{*}\left(B^{\prime \prime}|\eta|\right)=\Omega\left(B^{\prime \prime}|\eta|\right)
$$

for some $B^{\prime}, B^{\prime \prime}>0$, where $N^{\#}$ is the associated function of $p ! / M_{p}$ and $N^{*}$ is the Young conjugate of the associated function of $N_{p}$. Thus, we have

$$
|\varphi(\xi+i \eta)| \leq C_{1} \exp \left[-M(|\xi| / A)+\Omega\left(B^{\prime \prime}|\eta|\right)\right],
$$

which completes the proof.

Remark. We can prove the characterization theorem for $W_{M}$ and $W^{\Omega}$ similarly. 


\section{REFERENCES}

[1] J. Chung, S. Y. Chung, and D. Kim, Une caractérisation de l'espace de Schwartz, C. R. Acad. Sci. Paris Sér. I Math. 316 (1993), 23-25. MR 93m:46040

[2] _ A characterization for Fourier hyperfunctions, Publ. RIMS, Kyoto Univ. 30 (1994), 203-208. MR 94m:46066

[3] _ Equivalence of the Gelfand-Shilov spaces, J. Math. Anal. Appl., (to appear).

[4] S.-Y. Chung and D. Kim, Representation of quasianalytic ultradistributions, Ark. Mat. 31 (1993), 51-60. MR 94e:46069

[5] S. Y. Chung, D. Kim, and S. K. Kim, Equivalence of the spaces of ultradifferentiable functions and its application to the Whitney extension theorem, Rend. Mat. Appl. VII 12 (1992), 365380. MR 93k: 46030

[6] I. M. Gelfand and G. E. Shilov, Generalized functions II, III, Academic Press, New York and London, 1967. MR 55:8786c

[7] L. Hörmander, A uniqueness theorem of Beurling for Fourier transform pairs, Ark. Mat. 29 (1991), 237-240. MR 93b:42016

[8] A. I. Kashpirovsky, Equality of the spaces $S_{\alpha}^{\beta}$ and $S_{\alpha} \cap S^{\beta}$, Functional Anal. Appl. 14 (1978), 60.

[9] H. Komatsu, Ultradistributions I, J. Fac. Sci. Univ. Tokyo Sect. IA Math. 20 (1973), 25-105. MR 47:9277

Department of Mathematics, Kunsan National University, Kunsan 573-360, Korea

E-mail address: jychung@knusun1.kunsan.ac.kr

Department of Mathematics, Sogang University, Seoul 121-742, Korea

E-mail address: sychung@ccs.sogang.ac.kr

Department of Mathematics, Seoul National University, Seoul 151-742, Korea

E-mail address: dhkim@math.snu.ac.kr 